\title{
Questões fonoaudiológicas e odontológicas na percepção de idosos que acessam serviços do Sistema Único de Saúde
}

\author{
Speech therapy and oral health issues in the perception of the older adults that \\ access the Unified Health System
}

\author{
Ewelyn de Freitas Farias ${ }^{1}$ (D), Patricia Tavora Bulgarelli ${ }^{2}$ (D), Rafaela Soares Rech ${ }^{1}$ (D), Luana Xavier Marques ${ }^{1}$ (D), \\ Camila Mello dos Santos ${ }^{1,2}$ (D), Alexandre Fávero Bulgarelli ${ }^{1,2}$ (D)
}

\begin{abstract}
RESUMO
Modelo de estudo: Estudo observacional e transversal com dados coletados a partir de questionários aplicados aos idosos em visitas domiciliares, sobre acesso a serviços de saúde. Objetivo: Analisar a percepção de idosos independentes sobre questões fonoaudiológicas e odontológicas nos serviços de Atenção Primária à Saúde do Sistema Único de Saúde. Métodos: Foram analisadas frequências absolutas das variáveis de estudo relacionadas a questões fonoaudiológicas e odontológicas através do teste do Qui-quadrado e Teste Exato-de-Fisher, ambos com nível de 0,05\% de significância. Resultados: Os idosos entrevistados foram predominantemente mulheres $(76,5 \%)$, cor branca $(73,5 \%)$, com no mínimo ensino médio de escolaridade $(64,7 \%)$. Destes idosos, $92,6 \%$ afirmaram não possuir dificuldades para se alimentar, apesar de 51,5\% nunca ter feito uma avaliação fonoaudiológica. Houve maior proporção indicando que os idosos não necessitaram de consulta com fonoaudióloga (73,5\%). Quanto à utilização de serviços da atenção primária, 68,7\% utilizavam o sistema público e desses, 94,1\% autoperceberam sua condição de saúde bucal como regular/ruim. Conclusão: Sugere-se o fortalecimento do cuidado dos idosos de forma interdisciplinar e integral, a fim de abranger as necessidades e especificidades dessa população. A baixa percepção da importância do cuidado sugere a necessidade do fortalecimento dos serviços de fonoaudiologia para a saúde da população idosa brasileira.
\end{abstract}

Palavras-Chave: Atenção Primária à Saúde; Saúde do Idoso; Fonoaudiologia; Sistema Único de Saúde; Odontogeriatria.

\begin{abstract}
Study design: Cross-sectional study with data collected from questionnaires applied to older adults at domiciliary visits regarding access to health services. Objective: Analyzing the perception of independent elderly about speech-language and dental issues in the Primary Health Care Services of the Unified Health System/SUS. Methods: Absolute frequencies of the study variables related to speech-language and dental questions were analyzed using the Chi-square test and Fisher's Exact Test, both with a $0.05 \%$ significance level. Results: The elderly interviewed were predominantly women $(76.5 \%)$, white $(73.5 \%)$, with at least high school education $(64.7 \%) .0$ these, $92.6 \%$ said they had no difficulty in eating, although $51.5 \%$ never had a speech therapist evaluation. There was also a higher proportion indicating that the elderly did not need an appointment with the speech therapist (73.5\%). Regarding the use of primary care services, $68.7 \%$ used the public system, and of these, $94.1 \%$ self-perceived their oral health condition as fair/poor. Conclusion: It is suggested to strengthen the care of the elderly in an interdisciplinary and integral way to cover the needs and specificities of this population. The low perception of the importance of care suggests the need for strengthening speech therapy services for the health of the elderly Brazilian population.
\end{abstract}

Keywords: Primary Health Care; Health of the Elderly; Speech, Language and Hearing Sciences; Unified Health System; Geriatric Dentistry.

1. Centro de Pesquisa em Odontologia Social, Faculdade de Odontologia, Universidade Federal do Rio Grande do Sul (UFRGS), Porto Alegre (RS), Brasil.

2. Programa de Pós-Graduação em Saúde Coletiva, Escola de Enfermagem, Universidade Federal do Rio Grande do Sul (UFRGS), Porto Alegre (RS), Brasil.

$\triangle$ Alexandre Fávero Bulgarelli. Faculdade de Odontologia UFRGS - Rua Ramiro Barcelos, 2492 - Santa Cecilia. CEP: 90035-003.

Porto Alegre (RS), Brasil. alexandre.bulgarelli@ufrgs.br | Recebido em: 20/11/2019 | Aprovado em: 19/02/2020 


\section{INTRODUÇÃO}

O panorama brasileiro apresenta crescente aumento na proporção de idosos em relação à população total. Tal fato ocasiona o envelhecimento populacional no Brasil, caracterizando uma evidente mudança na pirâmide etária brasileira ${ }^{1}$. Avanços na Fonoaudiologia, Odontologia, Medicina e outras áreas da saúde, ao longo dos últimos anos, tornaram possível prevenir e curar muitas das doenças fatais e incapacitantes, assim como refletem em uma nova perspectiva populacional com aumento da expectativa de vida. Esta mudança da população reflete em novos desafios e revela a necessidade de representantes governamentais, pesquisadores científicos e líderes sociais se coordenarem diante das demandas epidemiológicas do novo perfil demográfico ${ }^{2}$. Um dos desafios é a implementação de modelos de atenção à saúde do idoso que se estruturem com as realidades dos municípios ${ }^{3}$. Este perfil engloba questões diagnósticas e reabilitadoras importantes e que merecem ser elucidadas nos serviços de saúde, seguindo os princípios e os preceitos do Sistema Único de Saúde ${ }^{4}$.

A qualidade funcional global da comunicação, da audição, da fala, da mastigação e deglutição, bem como a saúde bucal são compostas por condições objetivas (biológicas) e subjetivas (psicológicas), que possibilitam ao indivíduo exercer funções mecânicas como mastigação (consequente deglutição) e fala, sentir-se bem com sua aparência e relacionar-se socialmente. A qualidade funcional global oferece bem-estar, contribuindo para uma melhor qualidade de vida55.7 As condições que compõem essa qualidade global são funções vitais dos seres humanos e proporcionam sentido as suas vidas ${ }^{2}$.

A presença de doenças crônicas nos idosos é algo comum, mas apenas a sua ausência não é suficiente para os considerar saudáveis. A concepção de saúde vai além da ausência de comorbidades, mas na autonomia, interação social, capacidade de lazer e manutenção de suas atividades. As dificuldades de mastigação, fala e uma condição estética prejudicada pelas perdas dentárias podem provocar isolamento social do idoso, reclusão e problemas psicológicos como a depressão $0^{8,9}$ e até mesmo discriminação nos serviços de saúde ${ }^{10}$.

A saúde bucal da população merece atenção especial pelo fato de que, historicamente, os servi- ços odontológicos não possuíam como prioridade a atenção aos idosos. Deste modo, tal descaso reflete em uma população idosa com altos níveis de edentulismo e alta prevalência de cárie e de doenças periodontais ${ }^{11}$. Desde a origem dos serviços odontológicos no Brasil, percebe-se um direcionamento ao cuidado dos mais jovens. O primeiro modelo de atenção à saúde bucal brasileiro, o modelo incremental, foi definido como o modelo da Odontologia Sanitária que foi reduzida aos programas escolares, sendo ineficaz porque transformou-se em um padrão de atendimento, desconsiderando o contexto social e sem um olhar crítico epidemiológico da sociedade brasileira o qual já necessitava de cuidados mais amplos ${ }^{5}$. Quanto às questões de saúde no campo da fonoaudiologia, cabe destacar que dificuldade mastigatória acarreta queixas de deglutição e declínio da qualidade de vida dos idosos ${ }^{12,13}$.

Diante deste cenário, o objetivo do presente estudo é analisar a percepção de idosos robustos e independentes sobre questões envolvendo a Fonoaudiologia e a Odontologia e caracterizar o acesso a estes serviços de saúde na cidade de Porto Alegre/RS.

\section{MATERIAL E MÉTODO}

Trata-se de um estudo analítico transversal de base populacional, realizado por inquérito populacional com usuários o Sistema Único de Saúde/SUS em três Gerenciais Distritais/GD da cidade de Porto Alegre/RS, no período de setembro de 2016 a maio de 2017. Os dados foram coletados por aplicação de um questionário em entrevistas domiciliares sobre o acesso aos serviços básicos de saúde, incluindo serviços fonoaudiológicos e odontológicos no município. A amostra de estudo $(n=99)$ foi calculada respeitando-se o número da população do município, $42,4 \%$ atributo acesso em Porto Alegre - PCATool 14,15 , bem como 1,5 Deff - resguardado precisão (20\% eventuais recusas) e aplicação de filtros no banco de dados (Figura 1). Os filtros aplicados referem-se às respostas positivas para perguntas feitas durante 0 inquérito e que permitiram a análise dos dados com usuários que utilizavam todos os serviços disponibilizados nas unidades básicas de saúde do SUS, bem como usuários destes serviços do SUS juntamente com serviços privados de fonoaudiologia, das gerências/regiões estudadas. 


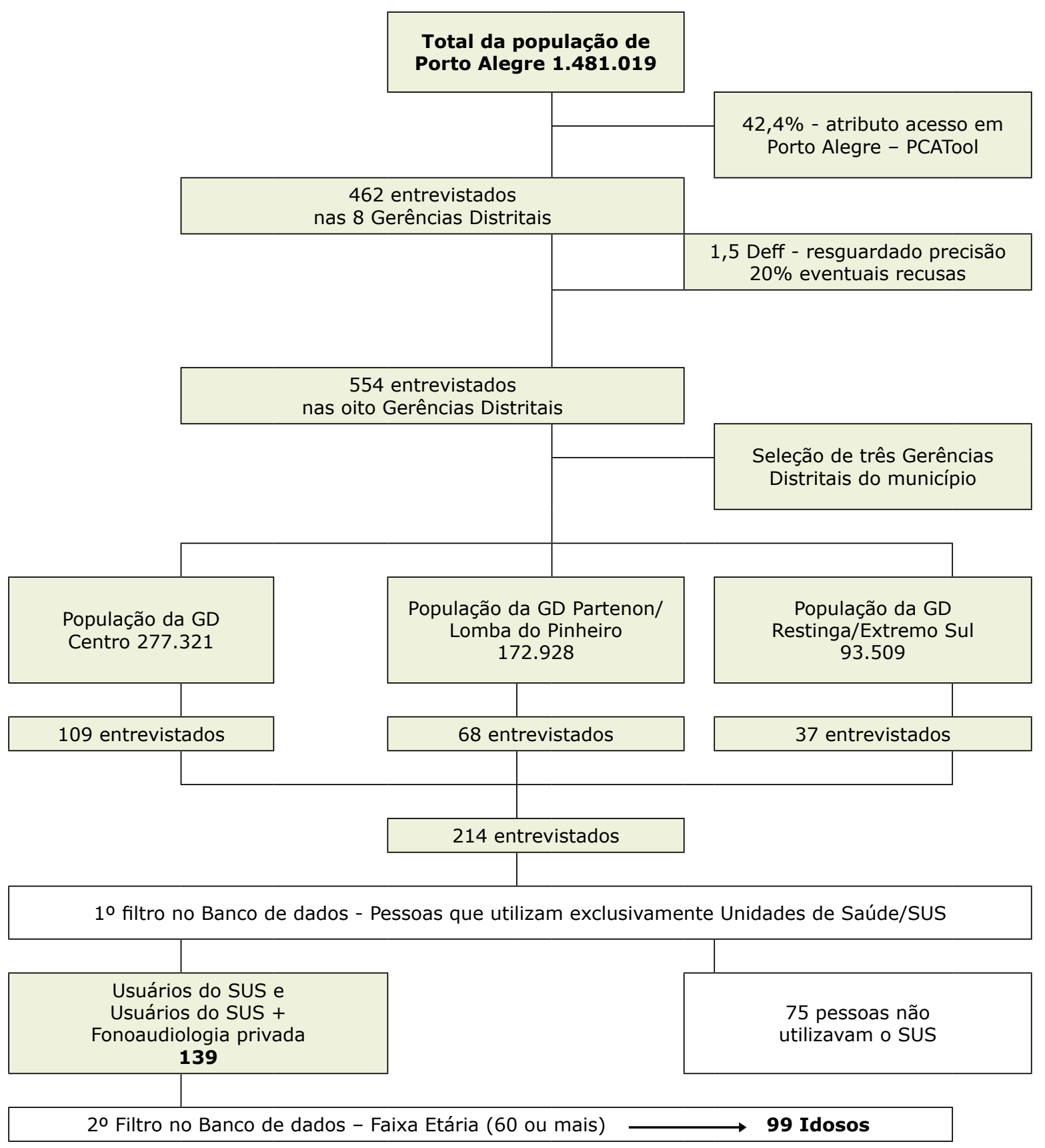

Figura 1 - Fluxograma da amostra para usuários idosos que utilizam os serviços de saúde na Atenção Primária à Saúde no SUS. Porto Alegre, 2019.

Foram selecionadas as três regiões com maior proporção de idosos no momento da coleta dos dados. Tais filtros foram: Filtro 1 "O senhor(a) utiliza serviços ofertados nas unidades básicas de saúde do SUS da região em que reside? Respostas Sim (apenas SUS ou SUS mais fonoaudiologia privado) ou Não; e o Filtro 2 Faixa Etária de 60 anos ou mais (Figura 1 ).
Os dados foram analisados no software SPSS v.21 (Chicago: SPSS Inc). Análises das frequências absolutas e relativas foram calculadas. $O$ teste do Qui-quadrado foi utilizado para avaliar as associações entre as variáveis estudadas segundo as características do atendimento (SUS ou SUS + Fonoaudiologia Privado), e quando este violou seus pressupostos utilizou-se o Teste Exato de Fisher, 
ambos com nível de 0,05\% de significância. O desfecho estudado é o acesso aos serviços de atenção primária à saúde do município de Porto Alegre/ RS. Em associação ao desfecho, foram investigadas variáveis sociodemográficas (sexo, raça/cor, escolaridade e situação conjugal), variáveis abordando questões fonoaudiológicas bem como odontológicas com distinção entre acesso ao serviço público e acesso ao serviço privado. As variáveis em estudo foram: realização de alguma avaliação de audição; necessidade de consulta fonoaudiológica; e dificuldade para se alimentar. Envolvendo as questões fonoaudiológicas foram utilizadas as perguntas: O senhor(a) já passou por alguma avaliação de sua audição? (sim; não) O senhor(a) sente que necessita consultar um fonoaudiólogo?(sim; não). O senhor(a) sente alguma dificuldade para se alimentar? (sim; não). Para o estudo das questões odontológicas utilizou-se as variáveis: Autopercepção da saúde bucal; Dificuldade para se alimentar; Utilização de alguma prótese dentária; Motivo da consulta odontológica (limpeza; outros), e última consulta odontológica nos últimos 12 meses. Para tanto, foram realizadas as seguintes perguntas: Como o senhor(a) acredita que está sua saúde bucal? (Boa, regular ou ruim); O senhor(a) utiliza algum tipo de prótese dentária? (sim, não); Por que motivo o senhor(a) foi ao dentista a última vez? (Limpeza, outro motivo); O senhor(a) consultou um dentista no último ano? (sim; não).

Este estudo foi aprovado pelo Comitê de Ética em Pesquisa da Secretaria Municipal de Saúde de Porto Alegre/RS (parecer 1.670.384) e do Comitê de Ética em Pesquisa da Universidade Federal do Rio Grande do Sul (Parecer n. 1.716.586). Os participantes foram informados quanto aos objetivos da pesquisa e solicitados a ler e assinar o Termo de Consentimento Livre e Esclarecido antes da aplicação do questionário de coleta de dados.

\section{RESULTADOS}

Do total da população estudada $(n=99)$, $68(68,7 \%)$ idosos utilizam apenas os serviços de saúde do SUS e $31(31,3 \%)$ idosos acessam os serviços do SUS e também serviços privados de Fonoaudiologia. Destes usuários exclusivos do SUS, houve o predomínio de idosos do sexo feminino 52 (76,5\%) e de cor branca 50 (73,5\%). Em relação à escolaridade, a maior proporção foi de idosos com, no mínimo, o ensino médio 44 (64,7\%). Já em relação a situação conjugal a maior proporção foi de idosos solteiro, separado ou viúvo 40 (58,8\%) (Tabela 1$)$.

\section{Tabela 1}

Características sociodemográficas e do uso de serviços fonoaudiológicos e odontológicos por idosos (n.99) do município de Porto Alegre no ano de 2017.

\begin{tabular}{llccc}
\hline \multirow{2}{*}{ Variáveis } & & \multicolumn{2}{c}{ Acesso serviços de saúde } \\
\cline { 3 - 4 } & & SUS & SUS + Fonoaudiologia Privado & $p$-value \\
\hline Sexo & Masculino & $16(23,5 \%)$ & $9(29,0 \%)$ & 0,364 \\
Raça & Feminino & $52(76,5 \%)$ & $22(71,0 \%)$ & 0,441 \\
& Branca & $50(73,5 \%)$ & $24(77,4 \%)$ & $7(22,6 \%)$ \\
Escolaridade & Não Branca & $18(26,5 \%)$ & $9(29,0 \%)$ & 0,248 \\
& Ensino fundamental & $24(35,3 \%)$ & $22(71,3 \%)$ & 0,438 \\
Situação conjugal & Ensino médio ou mais & $44(64,7 \%)$ & $14(45,2 \%)$ & $17(54,8 \%)$ \\
& Casado & $28(41,2 \%)$ & $\mathbf{3 1 ( 3 1 , 3 \% )}$ & \\
\hline
\end{tabular}

Ao observar as questões fonoaudiológicas percebeu-se que não houve associação significativa com o desfecho e quase a metade dos idosos que utilizam o SUS ainda não passou por uma avaliação de audição 35 (51,5\%). Notou-se, tam- bém, que houve maior proporção de idosos que não percebem a necessidade de consultar com um fonoaudiólogo $50(73,5 \%)$ e $63(92,6 \%)$ declararam não apresentar dificuldade para se alimentar (Tabela 2). 


\section{Tabela 2}

Características de questões fonoaudiológicas e o uso de serviços de saúde acessado por idosos (n.99) no município de Porto Alegre no ano de 2017.

\begin{tabular}{|c|c|c|c|c|c|}
\hline & \multirow{2}{*}{ Variáveis } & & \multicolumn{2}{|c|}{ Acesso a serviços de saúde } & \multirow{2}{*}{$p$-value } \\
\hline & & & SUS & SUS + Fonoaudiologia privado & \\
\hline \multirow{7}{*}{ 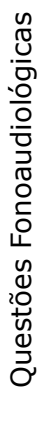 } & \multirow{2}{*}{ Avaliação de audição } & Sim & $33(48,5 \%)$ & $20(64,5 \%)$ & \multirow{2}{*}{0,103} \\
\hline & & Não & $35(51,5 \%)$ & $11(35,5 \%)$ & \\
\hline & \multirow{2}{*}{ Necessidade consulta } & Sim & $18(26,5 \%)$ & $11(35,5 \%)$ & \multirow{2}{*}{0,248} \\
\hline & & Não & $50(73,5 \%)$ & $20(64,5 \%)$ & \\
\hline & \multirow{3}{*}{ Dificuldade para se alimentar } & Sim & $5(07,4 \%)$ & $2(06,5 \%)$ & \multirow{2}{*}{0,619} \\
\hline & & Não & $63(92,6 \%)$ & $29(93,5 \%)$ & \\
\hline & & & $68(68,7 \%)$ & $31(31,3 \%)$ & \\
\hline
\end{tabular}

Tabela 3

Características de questões odontológicas e o uso de serviços de saúde acessado por idosos (n.99) no município de Porto Alegre no ano de 2017.

\begin{tabular}{|c|c|c|c|c|c|}
\hline & \multirow{2}{*}{ Variáveis } & & \multicolumn{2}{|c|}{ Acesso a serviços de saúde } & \multirow{2}{*}{$p$-value } \\
\hline & & & SUS & SUS + Fonoaudiologia Privado & \\
\hline \multirow{9}{*}{ 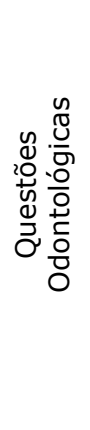 } & \multirow{2}{*}{ Autopercepção da saúde bucal } & Boa & $4(05,9 \%)$ & $5(16,1 \%)$ & \multirow{2}{*}{0,105} \\
\hline & & Regular/Ruim & $64(94,1 \%)$ & $26(83,9 \%)$ & \\
\hline & \multirow{2}{*}{ Utilização de prótese dentária } & Sim & $62(91,2 \%)$ & $18(58,1 \%)$ & \multirow{2}{*}{$<0,001$} \\
\hline & & Não & $6(8,8 \%)$ & $13(41,9 \%)$ & \\
\hline & \multirow{2}{*}{ Motivo da consulta } & Limpeza & $29(42,4 \%)$ & $21(67,7 \%)$ & \multirow{2}{*}{0,017} \\
\hline & & Outros & $39(57,6 \%)$ & $10(32,3 \%)$ & \\
\hline & \multirow{3}{*}{ Última consulta (12 meses) } & Sim & $36(53,0 \%)$ & $23(74,2 \%)$ & \multirow{3}{*}{0,038} \\
\hline & & Não & $32(47,0 \%)$ & $8(25,8 \%)$ & \\
\hline & & & $68(68,7 \%)$ & $31(31,3 \%)$ & \\
\hline
\end{tabular}

Em relação as questões odontológicas, maioria dos idosos que utilizam o SUS autopercebem a sua saúde bucal como regular/ruim $64(94,1 \%$ ) (Tabela 3 ). Dentre estes idosos que utilizam o SUS, a maioria utiliza algum tipo de prótese dentária (prótese de um ou mais elementos, prótese removível, prótese total superior e inferior) $62[(91,2 \%) p<0,001]$, e dentre os que frequentam também o serviço de fonoaudiologia privado apenas $18(58,1 \%)$ utilizam prótese. Ainda neste contexto, 36 [(53,0\%) $p<0,038]$ usuários do SUS relataram que realizaram a última consulta odontológica nos últimos 12 meses no SUS e dentre os que utilizam o serviço de fonoaudiologia privado 23 (74,2\%) (Tabela 3 ).

\section{DISCUSSÃO}

O presente estudo observou as percepções de idosos independentes e robustos que acessam serviços de atenção primária à saúde do município de Porto Alegre/RS. Tais idosos são aqueles capazes de gerenciar sua vida de forma independente e autônoma e não apresentam condições crônicas que caracterizam fragilidade e vulnerabilidade ${ }^{16}$. Nesta perspectiva, $68,7 \%$ destes idosos utilizam o SUS e $31,3 \%$ acessam a atenção primária por meio do serviço privado. Estudo nacional realizado com população de idosos aponta dados divergentes ao presente estudo, mostrando que a maioria dos idosos 
estudados acessavam serviços de odontológicos privados $(72,4 \%)^{17}$.

No Brasil, existem políticas públicas voltadas à atenção às necessidades e problemas dos idoso, porém, há muito trabalho a ser feito para que o SUS contemple as demandas desta população. O facilitado acesso aos serviços de saúde, adequação do modelo de atenção, e a prevalência significativa de problemas mentais e cognitivos e diferentes graus de dependência, merecem especial atenção ${ }^{18}$.

O presente estudo apontou que, proporcionalmente, mais mulheres são usuárias exclusivas dos serviços do SUS bem como idosos com menor escolaridade. Uma revisão de literatura sobre acesso a serviços de saúde bucal aponta semelhança em relação a variável sexo feminino, porém, certa diferença em relação a escolaridade sendo que o acesso esteve associado a maior escolaridade ${ }^{19}$.

Nunes, et al. (2017) ${ }^{19}$, apontam que a dor foi o maior motivo pela procura de serviços odontológicos no SUS por idosos, o que se diferencia dos achados do presente estudo que mostra que o maior motivo pela busca de dentista no SUS foi a prevenção. Este é um resultado relevante, pois sinaliza a necessidade e a importância do profissional da odontologia ter um olhar mais ampliado e acurado em relação à manutenção da saúde bucal do idoso de modo que, na busca pela prevenção em um acompanhamento regular, o dentista possa indagar questões como a qualidade da mastigação, da deglutição e da alimentação deste idoso e com isso, encaminhá-lo para os cuidados de um fonoaudiólogo(a). Questões fonoaudiológicas e odontológicas são fundamentais para prevenção de alterações na deglutição ${ }^{20,21}$. O cuidado interdisciplinar, o trabalho em equipe, e o compartilhamento do saber são essenciais para o diagnóstico e construção de um cuidado integral à saúde de um idoso com disfagia ${ }^{22,23}$.

Com o avançar da idade é evidenciado que algumas funções tornam-se mais prejudicadas, principalmente as funções estomatognáticas, como a perda da capacidade de controle do bolo alimentar e da coordenação motora na laterização deste durante a mastigação, a força mastigatória é reduzida e na trituração de alimentos, no caso dos edêntulos, realizam apenas um amassamento diante da sua condição ${ }^{24}$. A qualidade da mastigação é importante porque antecede a deglutição, que é uma função importante para a nutrição do indiví- duo. Quando a mastigação é alterada, pode ocorrer a disfagia, a dificuldade de engolir os alimentos. A dificuldade de mastigar e deglutir pode levar o idoso a restringir a sua alimentação a alimentos menos nutritivos, perda do prazer de se alimentar, quadros de perda de peso, desidratação, pneumonias aspirativas e anemias ${ }^{12,13}$.

A fala também pode ser afetada por problemas no aparelho estomatognático. A fala e a audição estão relacionadas a capacidade de interação social dos idosos, interferindo na sua qualidade de vida ${ }^{9}$. Modificações orgânicas do envelhecimento como calcificação e ossificação das estruturas laríngeas prejudicam a qualidade vocal do idoso; a perda gradativa da acuidade auditiva reflete na capacidade de socialização dos idosos ${ }^{25}$.

Além de sua função fisiológica para a nutrição, a deglutição envolve o contexto cultural da alimentação. Assim, problemas na deglutição comprometem, também, a socialização dos idosos acometidos por perdas dentárias e enfraquecimento do sistema estomatognático ${ }^{26}$. As principais alterações que ocorrem com o envelhecimento e que comprometem a deglutição são as perdas dentárias, aumento de tecido conjuntivo na cavidade bucal, redução da força mastigatória, redução na elevação anterior da laringe, acometendo principalmente as fases preparatória e oral da deglutição ${ }^{27}$. Nessa perspectiva, estudos apontam evidências de que a identificação precoce e reabilitação da disfagia orofaríngea por meio de reabilitação protética bucal é algo fundamental e relevante na melhora da deglutição em idosos. Em semelhança ao presente estudo, idosos relatam maior dificuldade em mastigar e deglutir alimentos duros ${ }^{17,20}$. Com o avançar da idade, ocorre um visível declínio no volume e capacidade na deglutição, como também um aumento no tempo médio de deglutição ${ }^{28}$. No presente estudo, $92,6 \%$ relataram que não apresentam dificuldade de se alimentar e apenas $07,4 \%$ afirmaram que sim, tem alguma dificuldade de deglutição. Sugere-se que o idoso, mesmo assintomático e sem queixas e com a referida autopercepção, deveria ser encaminhado para terapias fonoaudiológicas com o intuito de prevenção a disfagia e suas complicações.

Nas questões fonoaudiológicas, mesmo não havendo associação ao desfecho das variáveis estudadas, observou-se a busca pelos serviços privados. O fonoaudiólogo, lentamente vem ocupando um 
importante espaço nos serviços públicos de saúde incluindo a atenção básica e a alta complexidade ${ }^{29}$. Assim, o acesso ainda é restrito, o que faz com que a população busque o serviço privado. Percebe-se, deste modo, uma necessidade de ampliação de serviços fonoaudiológicos para idosos no SUS e este fato deve ser motivo de futuras discussões sobre integralidade do cuidado em saúde, visto que a perda auditiva e a dificuldade de mastigação e deglutição muitas vezes dificultam a integração social de idosos. Vale ressaltar que idosos atendidos no SUS indicam melhoras de habilidade auditivas após treinamentos auditivos computadorizados e utilização de próteses auditivas ${ }^{30-32}$.

Nas questões odontológicas, observou-se que a busca pelo dentista dentro de 12 meses não esteve associada ao desfecho ( $p$-valor $=0,038$ ), porém, pode-se afirmar que a maior proporção [59(60\%)] dos idosos visitou um dentista nos serviços ofertados pelo SUS nos últimos 12 meses, possivelmente pela oferta deste profissional no sistema público de saúde. Esta variável, visita ao dentista dentro dos últimos 12 meses, foi relatada na literatura com resultado contrário ao presente estudo, sendo que $63,2 \%$ dos idosos não haviam visitado o dentista ${ }^{17}$. Ao observar a questão da utilização de alguma prótese bucal em associação ao desfecho percebeu-se que a associação foi positiva. Idosos usuários de próteses acessam mais os serviços odontológicos do SUS.

Fatores odontológicos e fonoaudiológicos interferem na autopercepção de idosos sobre sua própria saúde e deste modo reflete na busca por diferentes serviços na atenção primária como no presente estudo. A literatura aponta que autopercepção de condições bucais ruins são mais prevalentes em idosos socialmente pró-ativos, com necessidades de tratamentos protéticos, em idosos em busca de meIhoria da sua qualidade de vida $17,33,34$. Nesta perspectiva de acesso a serviços e autopercepção, pode-se compreender que possivelmente muitos idosos não autopercebam as dificuldades mastigatórias, pois tendem a achar que é algo normal, assim como os problemas de deglutição. Deste modo, muitos deles não são diagnosticados e não procuram tratamento.

Mesmo com a limitação de ser um estudo que não permitiu afirmativas de causalidade, houve importantes associações que suscitam diversos questionamentos abrindo possibilidades para novos estudos. Acredita-se que o presente estudo trouxe importantes reflexões frente a resposta a seu objetivo o qual foi atingido.

\section{CONCLUSÃO}

O presente estudo apontou temas importantes por meio das análises propostas. Mostrou-se um estudo original, que por meio da análise de questões fonoaudiológicas e odontológicas concluiu que os idosos portadores de próteses dentárias buscam os serviços do SUS e em situação oposta, o acesso dos idosos aos serviços fonoaudiológicos acontece prioritariamente no sistema privado. No processo de autopercepção das condições bucais e fonoaudiológicas, sugere-se a busca por um fortalecimento do atendimento interdisciplinar ao idoso no SUS, visto que a maioria utiliza algum tipo de prótese bucal e necessita de diagnósticos precoces de problemas fonoaudiológicos.

O presente estudo é original em sua proposição, e suscita estudos com amostras mais abrangentes sobre estes questionamentos tão importante para a saúde dos idosos. Sugere-se a realização de estudos que apontem os motivos subjetivos que fazem com que os idosos não reconheçam e não busquem serviços de fonoaudiologia. O presente estudo também levanta a necessidade de aumentar a oferta de serviços de fonoaudiologia na Atenção Primária do SUS para ampliar o acesso a este serviço tão importante e necessário para a população idosa brasileira.

\section{CONFLITO DE INTERESSES}

Os autores declaram não haver conflito de interesses.

\section{FONTE DE FINANCIAMENTO}

Próprio.

\section{AGRADECIMENTOS}

Ao Centro de Pesquisa em Odontologia Social da Universidade Federal do Rio Grande do Sul pelo auxílio no financiamento da presente pesquisa. 


\section{REFERÊNCIAS}

1. Instituto Brasileiro de Geografia E Estatística - IBGE. Sinopse do Censo Demográfico de 2010. Rio de Janeiro, 2011.

2. Miranda GMD, et al. O envelhecimento populacional brasileiro: desafios e consequências sociais atuais e futuras. Revista Brasileira de Geriatria e Gerontologia, 2016, 19(3):507-519.

3. Coelho LP, Motta LBD, Caldas CP. Rede de atenção ao idoso: fatores facilitadores e barreiras para implementação. Physis: Revista de Saúde Coletiva, 2019, 28(1): e280404.

4. Brasil. Ministério da Saúde. Lei 8080 de 19 de setembro de 1990. Dispõe sobre as condições para a promoção, proteção e recuperação da saúde, a organização e o funcionamento dos serviços correspondentes e dá outras providências. Disponível em: http://www.planalto.gov. br/ccivil_03/leis/l8080.htm. Acesso em 07 out 2019.

5. Narvai PC. Saúde bucal coletiva: caminhos da odontologia sanitária à bucalidade. Rev. Saúde Pública, 2006, 40(1):141-147.

6. Narvai PC, Frazão P. Saúde bucal no Brasil: muito além do céu da boca. Rio de Janeiro: Fiocruz, 2008.

7. Carniel $\mathrm{CZ}$, et al. Implications of using the Hearing Aids on quality of life of elderly. Revista CoDAS, 2017,29(5). http://dx.doi.org/10.1590/2317-1782/20172016241

8. Brasil. Ministério da Saúde. Portaria no. 2.528/2006. Aprova a Política Nacional da Pessoa Idosa. Diário Oficial República Federativa do Brasil. 2006;237(20):4

9. Santiago LM, et al. Caracterização da saúde de idosos numa perspectiva fonoaudiológica. Rev. CEFAC, 2016,8(5):1088-1096.

10. Baumgarten A, Peron TB, Bastos JL, Toassi RFC, Hilgert JB, Hugo FN, Celeste RK. Experiências de discriminação relacionadas aos serviços de saúde: análise exploratória em duas capitais do Sul do Brasil. Epidemiologia e Serviços de Saúde, 2015,24:353-362. http://dx.doi. org/10.5123/S1679-49742015000300002.

11. Moreira RS, et al. A saúde bucal do idoso brasileiro: revisão sistemática sobre o quadro epidemiológico e acesso aos serviços de saúde bucal. Cad. Saúde Pública, 2005,21(6):1665-1675.

12. Souza A, Oda AL. A importância do levantamento de queixas de idosos institucionalizados durante a entrevista para o planejamento da reabilitação fonoaudiológica. O mundo da saúde, 2008;32(2):157-69.

13. Rodrigues LKV, Pernambuco L. Produção científica sobre disfagia orofaríngea em idosos nos periódicos brasileiros: uma análise bibliométrica. Distúrbios da Comunicação, 2017,29(3):529-538. https://doi.org/10.23925/ 2176-2724.2017v29i3p529-538

14. Harzheim E, et al. Consistência interna e confiabilidade da versão em português do Instrumento de Avaliação da
Atenção Primária (PCATool-Brasil) para serviços de saúde infantil. Cad Saúde Pública, 2006,22(8):1649-1659.

15. Bulgarelli PT, et al. A perspectiva do usuário sobre o acesso aos serviços da atenção primária à saúde. Tempus Actas de Saúde Coletiva. 2018,11(3):216-231.

16. Moraes EM, et al. Índice de Vulnerabilidade Clínico Funcional-20 (IVCF-20): reconhecimento rápido do idoso frágil. Rev. Saúde Pública, 2016,50(81). http://dx.doi. org/10.1590/s1518-8787.2016050006963

17. Milagres CS, et al. Self-perceived oral health status, chewing ability and longevity in the elderly. Ciencia \& saude coletiva, 2018,23(5):1495-1506. http://dx.doi. org/10.1590/1413-81232018235.14572016.

18. Martins $A B$. Atenção Primária a Saúde voltada as necessidades dos idosos: da teoria à prática. Ciência \& Saúde Coletiva, 2014,19:3403-3416.

19. Nunes CSR, et al. Acesso aos serviços de saúde bucal de adultos e idosos. Estação Científica (UNIFAP), 2017,7(3):09-18. http://dx.doi.org/10.18468/estcien.2017v7n3.p09-18

20. Rech RS, et al. Deglutição no envelhecimento e a odontologia. Revista da Faculdade de Odontologia-UPF, 2018, 23(1):77-83.

21. Quek HC, Lee YS. Dentistry considerations for the dysphagic patient: Recognition of condition and management. Proceedings of Singapore Healthcare, 2010105819868252. 2019

22. Peduzzi $M$, et al. Trabalho em equipe na perspectiva da gerência de serviços de saúde: instrumentos para a construção da prática interprofissional. Physis: Revista de Saúde Coletiva, 2011,21:629-646.

23. Farpour S, Farpour H, Zakeri M. Oropharyngeal Dysphagia in Elderly People. Journal of Rehabilitation Sciences \& Research, 2018,5(4):120-123. 10.2147/CIA.S97481

24. Oliveira BS, Delgado SE, Brescovici SM. Alterações das funções de mastigação e deglutição no processo de alimentação de idosos institucionalizados. Revista brasileira de geriatria e Gerontologia, 2014,17(3):575-587.

25. Soares EB, et al. Hábitos vocais em dois grupos de idosos. Rev CEFAC. 2007,9(2):221-227.

26. Marcolino J. Achados fonoaudiológicos na deglutição de idosos do município de Irati - Paraná. Revista Brasileira de Geriatria e Gerontologia, 2009, 12:193-200.

27. Garcia MAA. Idosos em cena: falas do adoecer. Interface, 2005, 9(51):537-52.

28. Moreira GMM, Pereira SRM. Desempenho de idosos brasileiros no teste de deglutição de $100 \mathrm{ml}$ de água. Rev Soc Bras Fonoaudiol, 2012, 17(1):9-14.

29. Relly $C D$, et al. Atuação fonoaudiológica no sistema público de saúde $-r$ evisão de literatura. Journal of health (FJH), 2019,1(1):212-231. 
30. Teixeira TS, Costa-Ferreira MID. Computer-based auditory training for elderly with hearing aids provided by the Health Care System. Audiology-Communication Research, 2018, 23:e1786. http://dx.doi.org/10.1590/23176431-2016-1786

31. Picinini TDA, et al. Restriction in social participation and satisfaction with hearing aids-a study on post-fitting. Audiology-Communication Research, 2017,22(1). http://dx.doi.org/10.1590/2317-6431-2016-1830
32. Correia RO, et al. Reabilitação auditiva por aparelhos de amplificação sonora individual (AASI): perfil epidemiológico de pacientes adaptados em um hospital terciário em 5 anos. Revista de Medicina da UFC. 2017,57(2):26-30.

33. Lima LHMA, et al. Autopercepção oral e seleção de alimentos por idosos usuários de próteses totais. Rev Odontol UNESP, 2007, 36(2):131-6.

34. Nunes $\mathrm{CI}$, Abegg C. Factors associated with oral health perception in older Brazilians. Gerodontology, 2008, 25(1):42-8. 
